\title{
A REGRESSION PROBLEM CONCERNING STATIONARY PROCESSES
}

BY

\author{
M. P. HEBLE
}

1. Introduction. Let $y(t)=x(t)+m(t)$ be a continuous parameter stochastic process observed for $0 \leqq t \leqq T$, the mean value being $m(t)=E y(t)$, and $x(t)$ being weakly stationary (see $[7$, p. 33]) and continuous in the mean, with $E x(t) \equiv 0$. The means $m(t)$ are assumed to be of the form

$$
m(t)=\gamma_{1} \phi_{1}(t)+\gamma_{2} \phi_{2}(t)+\cdots+\gamma_{p} \phi_{p}(t)
$$

where $\gamma_{1}, \cdots, \gamma_{p}$ are unknown parameters and $\phi_{1}(t), \cdots, \phi_{p}(t)$ are known continuous functions of $t$. We want good linear unbiased estimates

$$
c=\left(\begin{array}{c}
c_{1} \\
\vdots \\
c_{p}
\end{array}\right) \quad \text { of } \quad \gamma=\left(\begin{array}{c}
\gamma_{1} \\
\vdots \\
\gamma_{p}
\end{array}\right) .
$$

One criterion used is the "naive" criterion of "least squares," viz. minimizing the expression

$$
\int_{0}^{T}\left|y(t)-\sum_{j=1}^{p} c_{j} \phi_{j}(t)\right|^{2} d t
$$

with respect to $c$. The integral involving $y(t)$ is here understood to be in the sense of Cramér [3, Lemma 3]. Another criterion used is that of "minimum variance," i.e. minimizing the error-covariance matrix $E(c-\gamma)(c-\gamma) *$ with respect to $c$.

The object of this paper is to derive, under certain assumptions, asymptotic expressions (following Grenander and Rosenblatt) for the error-matrices of the least-squares and the minimum variance estimates, $c_{L, T}$ and $c_{M, T}$, respectively, i.e. the expressions

$$
\begin{aligned}
& \lim _{T \rightarrow \infty} D_{T} E\left(c_{L, T}-\gamma\right)\left(c_{L, T}-\gamma\right)^{*} D_{T}, \\
& \lim _{T \rightarrow \infty} D_{T} E\left(c_{M, T}-\gamma\right)\left(c_{M, T}-\gamma\right)^{*} D_{T}
\end{aligned}
$$

where the $D_{t}$ are suitable non-negative definite norming matrices (see [ 7 , p. 235]). Following Grenander and Rosenblatt we shall say that $c_{L, T}$ is asymptotically efficient if $(1.1)=(1.2)$.

Our object is further to investigate whether a necessary and sufficient

Received by the editors May 23, 1960. 
condition for the asymptotic efficiency of $c_{L, T}$ can be derived, analogous to one obtained by Grenander and Rosenblatt for the discrete-parameter case (see [7, pp. 86-88, 233-245]).

The problem can be regarded as purely a problem of approximations. The plan adopted in this paper is that of approximating by a sequence of discrete parameter situations to the continuous parameter situation. Let $S=\left\{t_{1}, t_{2}, \cdots\right\}$ be any denumerable dense set of time points in $(0, T)$. Let $S_{k}=\left\{t_{1}, t_{2}, \cdots, t_{k}\right\}$, and $D_{k}$ be the set of observations $\left\{y_{t_{i}}\right\}, i=1, \cdots, k$; $k=1,2, \cdots$. So $D_{k} \subseteq D_{k+1}$ for all $k$. For each $D_{k}$ consider the corresponding values of the regression functions $\phi_{i}\left(t_{j}\right), i=1, \cdots, p ; j=1, \cdots, k$; as also the "least squares" and "minimum variance" estimates $c_{L, S_{k}}, c_{M, S_{k}}$ (cf. [7, pp. 86-88]), their respective error-matrices being denoted by $e_{S_{k}}, E_{S_{k}}$. Also denote the sample $\left\{y_{t}\right\}, 0 \leqq t \leqq T$, by $D$, the corresponding least squares and minimum variance estimates by $c_{L, T}, c_{M, T}$ respectively, and their respective errors by $e_{T}, E_{T}$. The estimates $c_{L, T}, c_{M, T}$ have yet to be defined. The next thing to prove is that (at any rate for a suitable choice of the dense denumerable set $S$ ) $c_{L, S_{k}}, c_{M, S_{k}}$ converge to $c_{L, T}, c_{M, T}$ respectively (in the mean square), and their respective errors converge to $e_{T}, E_{T}$.

The error-matrix $E_{T}$ will be shown to be nonsingular and continuous under certain conditions (Theorem $1, \S 3$ ).

These conditions will be shown to be satisfied in the case of certain purely nondeterministic stationary processes (the "THGM $\mathrm{T}_{\mathrm{N}}$ " processes of Doob [5]), provided the regression functions are sufficiently smooth. It is interesting to note that in the case of these special processes, one can follow a direct procedure, mimicking a method due to Mann and Moranda [8], to show that $E_{T}$ is nonsingular and continuous. However this last computation will be omitted here.

Our assumptions for the final results are the following: the $x(t)$-process is continuous in the mean; the spectral distribution function of the $x(t)$-process is absolutely continuous, the spectral density $f(\lambda)$ being positive, continuous and of bounded variation on $(-\infty, \infty)$; the regression functions $\phi(t)$ satisfy certain conditions analogous to those imposed by Grenander and Rosenblatt in the discrete parameter case (see $\S 5$ ); the integral operator $R$ on $L^{2}(0, T)$ with the continuous covariance function $r(t, \tau)$ for its kernel has an inverse $R^{-1}$ and that each of the regression functions $\phi$ is in the domain of $R^{-1}$.

For convenience, henceforth the assumptions stated in the last paragraph will be referred to as the conditions $\mathrm{A}$. With these assumptions, we first obtain asymptotic expressions (in the sense of GR) for the error-covariance matrices $e_{T}$ and $E_{T}$ (Theorem $2, \S 6$ ). With the same assumptions $\mathrm{A}$, we next obtain a necessary and sufficient condition for $c_{L, T}$ to be asymptotically efficient (Theorem $3, \S 7)$.

The class of regression functions mentioned above includes the following polynomial, trigonometric, and mixed polynomial-trigonometric regressions: 
(i)

$$
\begin{array}{rlrl}
\text { (i) } & \phi(t) & =t^{\nu}, & \nu \geqq 0 \text { an integer; } \\
\text { (ii) } & \phi(t)=t^{\nu} e^{i t \mu}, & \nu \geqq 0 \text { an integer; } \mu \text { any real no.; } \\
\text { (iii) } & \phi(t)=\left\{\begin{array}{ll}
t^{\nu} \cos t \mu \\
t^{\nu} \sin t \mu
\end{array}\right\}, & \nu \geqq 0 \text { an integer; } \mu \text { any real no. }
\end{array}
$$

2. The least squares estimate. We shall now define the "Least-Squares Estimate." Clearly

$$
\begin{aligned}
\int_{0}^{T} \mid y(t) & -\left.\sum_{j=1}^{p} c_{j} \phi_{j}(t)\right|^{2} d t \\
= & \int_{0}^{T}|y(t)|^{2} d t-\sum_{j=1}^{p} \bar{c}_{j} \int_{0}^{T} \overline{\phi_{j}(t)} y(t) d t \\
& \quad-\sum_{j=1}^{p} c_{j} \int_{0}^{T} \phi_{j}(t) \overline{y(t)} d t \quad \sum_{i, j=1}^{p} \bar{c}_{i} c_{j} \int_{0}^{T} \overline{\phi_{i}(t)} \phi_{j}(t) d t .
\end{aligned}
$$

The right side is equal to

$$
\int_{0}^{T} \mid\left(\left.y(t)\right|^{2}-c^{*} z-z^{*} c+c^{*} B c\right.
$$

where

$$
c=\left(\begin{array}{c}
c_{1} \\
\dot{\cdot} \\
c_{p}
\end{array}\right), \quad z=\left(\begin{array}{c}
z_{1} \\
\dot{z_{p}} \\
\cdot
\end{array}\right)=\left(\begin{array}{c}
\int_{0}^{T} \overline{\phi_{1}(t)} y(t) d t \\
\cdot \\
\int_{0}^{T} \overline{\phi_{p}(t)} y(t) d t
\end{array}\right),
$$

and $B=\left(\left(\int_{0}^{T} \overline{\phi_{i}(t)} \phi_{j}(t) d t\right)\right)$. The matrix $B$ is assumed to be strictly positive definite. Let $B^{1 / 2}$ be its positive definite square root. Then (2.1) is equal to

$$
\int_{0}^{T}|y(t)|^{2}+\left[B^{1 / 2} c-B^{-1 / 2} z\right]^{*}\left[B^{1 / 2} c-B^{-1 / 2} z\right]-z^{*} B^{-1} z
$$

We see that (2.2) is minimized for

$$
B^{1 / 2} c=B^{-1 / 2} z
$$

i.e.,

$$
c=B^{-1} z .
$$

Thus we conclude: The least squares estimate $=c_{L, T}=B^{-1} Z$. It is easily seen that $c_{L, T}$ is linear and unbiased. Its error 


$$
\begin{aligned}
& =e_{T}=E\left(c_{L, T}-\gamma\right)\left(c_{L, T}-\gamma\right)^{*} \\
& =B^{-1}\left(\left(\int_{0}^{T} \int_{0}^{T} \overline{\phi_{i}(t)} r(t-\tau) \phi_{j}(\tau) d t d \tau\right)\right) B^{-1}
\end{aligned}
$$

where $r(t-\tau)=E x(t \overline{x(\tau)}$.

Next define the sets $S_{k}$ as follows. Let $n$ be a fixed positive integer. For each positive integer $k$ define $\epsilon_{k}$ by: $2^{k-1} n \epsilon_{k}=T$, where $(0, T)$ is the interval over which the sample is observed. We take $S_{k}=\left\{0, \epsilon_{k}, 2 \epsilon_{k}, \cdots, T\right\}$ and the dense denumerable set $S$ is defined to be the union of all the sets $S_{k}$, $k=1,2, \cdots$.

We know that $[7, \mathrm{pp} .86-88]$ for $S_{k}$, the least squares estimate and its error are given by

$$
\begin{aligned}
C_{L, S_{k}} & =\left[\Phi_{S_{k}}^{*} \Phi_{S_{k}}\right]^{-1} \Phi_{S_{k} y_{S_{k}}}^{*}, \\
e_{S_{k}} & =\left[\Phi_{S_{k}}^{*} \Phi_{S_{k}}\right]^{-1} \Phi_{S_{k}}^{*} R_{S_{k}} \Phi_{S_{k}}\left[\Phi_{S_{k}}^{*} \Phi_{S_{k}}\right]^{-1}
\end{aligned}
$$

where $\Phi_{S_{k}}$ is the $(k \times p)$ matrix: $\Phi_{S_{k}}=\left(\left(\phi_{j}\left(t_{i}\right)_{j}, i=1, \cdots, k ; j=1, \cdots, p\right)\right)$, $R_{S_{k}}$ is the $(k \times k)$ matrix: $R_{S_{k}}=\left(\left(r\left(t_{i}-t_{j}\right) ; i, j=1, \cdots, k\right)\right)$, and $y_{S_{k}}$ is the $k-$ vector of observations:

$$
y_{S_{k}}=\left(\begin{array}{c}
y\left(t_{1}\right) \\
\vdots \\
y\left(t_{k}\right)
\end{array}\right)
$$

It is easy to see that as $k \rightarrow \infty, c_{L, S_{k}}$ converges to $c_{L, T}$ in the mean square, and $e_{S_{k}} \rightarrow e_{T}$.

3. The "Markov" or "minimum variance" estimate. Next, we shall consider the minimum variance criterion. The fact that $x(t)$ is continuous in the mean implies that the covariance function $r(t, \tau)$ is continuous. Now assume that for every finite sequence $S_{n}=\left\{t_{1}, t_{2}, \cdots, t_{n}\right\}$ of time points, all the inverse matrices occurring in the formal definition of the Markov estimate (cf. $\left[7\right.$, pp. 86-88]): $\left[\Phi_{S_{n}}^{*} R_{S_{n}}^{-1} \Phi_{S_{n}}\right]^{-1} \Phi_{S_{n}}^{*} R_{S_{n}}^{-1} y_{S_{n}}$, exist, where $\Phi_{S_{k}}$ is the $(k \times p)$ matrix $\left(\left(\phi_{j}\left(t_{i}\right) ; i=1, \cdots, k ; j=1, \cdots, p\right)\right), R_{S_{k}}$ is the $(k \times k)$ matrix $\left(\left(r\left(t_{i}-t_{j}\right) ; i, j=1, \cdots, k\right)\right)$, and $y_{S_{k}}$ is the $k$-vector

$$
\left(\begin{array}{c}
y\left(t_{1}\right) \\
\vdots \\
y\left(t_{k}\right)
\end{array}\right) .
$$

For the purposes of the present section, let $S=\left\{t_{1}, t_{2}, \cdots\right\}$ be any denumerable dense set of time points in $(0, T)$. Let $S_{k}=\left\{t_{1}, t_{2}, \cdots, t_{k}\right\}$ and $D_{k}$ be the sample $\left\{y_{t_{i}}\right\}, i=1, \cdots, k ; k=1,2, \cdots$. So $\mathfrak{D}_{k} \subseteq D_{k+1}$ for all $k$. We then know that (cf. [7, pp. 86-88]) for each $\mathfrak{D}_{k}$ there exists a unique mini- 
mum variance linear unbiased estimate (viz. the "Markov" estimate) $c_{M, S_{k}}$, with error

$$
E_{S_{k}}=\left[\Phi_{S_{k}}^{*} R_{S_{k}}^{-1} \Phi_{S_{k}}\right]^{-1}
$$

Clearly

$$
E_{S_{k}} \geqq E_{S_{k+1}} \geqq \cdots \geqq 0 .
$$

Hence there exists a limit matrix $E_{S} \geqq 0$ of the sequence $\left\{E_{S_{k}}\right\}$.

Next we show: The error covariance matrix of any linear unbiased estimate is $\geqq E_{\mathrm{S}}$.

Let $m^{*}$ be any linear unbiased estimate based on the sample $\{y(t)\}$, $0 \leqq t \leqq T$. Say

$$
m^{*}=\left(\begin{array}{c}
m_{1}^{*} \\
\vdots \\
\dot{m}_{p}^{*}
\end{array}\right) .
$$

Then, because of the continuity in the mean of the process, it is easy to show that $m^{*}$ is the limit in the mean square of a sequence $\left\{\mu_{k}^{*}\right\}$ of linear unbiased estimates based on finite sets of time-points, and we can assume these finite sets of time-points to be $S_{j_{k}}$, for some increasing sequence of integers $\left\{j_{k}\right\}$. Let the error-matrices of $\mu_{k}^{*}$ and $m^{*}$ be denoted by $\delta_{k}$ and $\delta$ respectively. Then

$$
\delta_{k} \geqq E_{S_{j_{k}}} \geqq E_{S} \text {. }
$$

Letting $k \rightarrow \infty$ we obtain

$$
\delta \geqq E_{S}
$$

From this we conclude: $E_{\mathrm{s}}$ is independent of the dense denumerable set $S:$ if $S^{\prime}$ is any other dense denumerable set, $E_{S}=E_{S^{\prime}}$. For by the preceding result $E_{S^{\prime}} \geqq E_{S}$. But by the same argument $E_{S} \geqq E_{S^{\prime}}$. Hence $E_{S^{\prime}}=E_{S}=E_{T}$ say.

Next: the estimates $\left\{c_{M, s_{m}}\right\}$ form a Cauchy sequence:

$$
E\left(c_{M, S_{m}}-c_{M, S_{n}}\right)\left(c_{M, S_{m}}-c_{M, S_{n}}\right)^{*} \rightarrow 0
$$

as $m, n \rightarrow \infty$. This is proved by using the matrix parallelogram law:

$$
\text { for all } p \text {-vectors } x \text { and } y \text {, }
$$

$$
E(x+y)(x+y)^{*}+E(x-y)(x-y)^{*}=2 E x x^{*}+2 E y y^{*} .
$$

We have only to take $x=m_{m} / 2, y=m_{n} / 2$ where we have set $m_{k}=c_{m, s_{k}}-\gamma$, and use the fact that the linear unbiased estimates of $\gamma$ form a convex set.

From this it follows next that the sequence $\left\{c_{M, s_{k}}\right\}$ tends to a limit $c_{M, s}$ in mean square. Clearly $E_{T}$ is the error-matrix of $c_{M, S}$.

Furthermore, the limit vector $c_{M, S}$ is independent of the dense denumerable 
set $S: c_{M, S}=c_{M, S^{\prime}}$ where $S, S^{\prime}$ are any two dense denumerable sets. For, write $m$ for $c_{M, S}-\gamma$, and $m^{\prime}$ for $c_{M, S^{\prime}}-\gamma$. Then using the convexity property of the set of linear unbiased estimates of $\gamma$ and the above parallelogram law (3.1), we easily obtain

$$
E\left(\frac{m+m^{\prime}}{2}\right)\left(\frac{m+m^{\prime}}{2}\right)^{*}=E_{T}
$$

and

$$
E_{T}+E\left(\frac{m-m^{\prime}}{2}\right)\left(\frac{m-m^{\prime}}{2}\right)^{*}=E_{T}
$$

Hence

$$
E\left(m-m^{\prime}\right)\left(m-m^{\prime}\right)^{*}=0 .
$$

This shows that $m=m^{\prime}$, i.e., $c_{M, S}=c_{M, S^{\prime}}=c_{M, T}$, say.

To sum up, we have proved:

LEMmA 1. There exists a unique linear unbiased estimate $c_{M, T}$ with minimal error-covariance matrix $E_{T}$. We shall call $c_{M, T}$ the Markov estimate based on the sample $\left\{y_{t}\right\}, 0 \leqq t \leqq T$.

For the final results of this paper it is essential that the error-matrix $E_{T}$ be nonsingular and continuous. We therefore want a reasonable condition which will ensure that $E_{T}$ will be nonsingular and continuous in $T$. The following theorem is to this end.

THEOREM 1. Suppose the conditions stated earlier at the beginning of this section hold, and suppose, for any $T>0$ the integral operator $R$ on $L^{2}(0, T)$ with the continuous covariance function $r(t, \tau)$ for its kernel has an inverse $R^{-1}$ and that each of the regression functions $\phi_{i}(t)$ is in the domain of $R^{-1}$. Then the error matrix $E_{T}$ of the Markov estimate will be nonsingular, and continuous in $T$.

Proof. Consider the matrix

$$
E_{T}=\lim _{N_{k} \rightarrow \infty}\left(\Phi_{N_{k}}^{*} R_{N_{k}}^{-1} \Phi_{N_{k}}\right)^{-1}
$$

It has been shown that the matrix

$$
\left(\Phi_{N_{k}}^{*} R_{N_{k}}^{-1} \Phi_{N_{k}}\right)^{-1}
$$

decreases as $N_{k}$ increases, i.e.,

$$
\Phi_{N_{k}}^{*} R_{N_{k}}^{-1} \Phi_{N_{k}}
$$


increases as $N_{k}$ increases. Since these error-matrices for the discrete situations are nonsingular (by virtue of earlier assumptions in the paper) it is enough to prove that with the assumptions of the proposition

$$
\lim _{N_{k} \rightarrow \infty} \Phi_{N_{k}}^{*} R_{N_{k}}^{-1} \Phi_{N_{k}} \text { is finite. }
$$

We shall show that the diagonal terms in this limit matrix are finite.

Consider a partition of $[0, T]$

$$
0=t_{0}<t_{1}<t_{2}<\cdots<t_{N_{k}}=T
$$

and suppose the subintervals $\Delta_{i}=\left(t_{i-1}, t_{i}\right)$ are of the same length $\delta=T / N_{k}$. Consider the integral operator $R_{N_{k}}$ with the piecewise constant kernel with values $R\left(t_{i}, t_{j}\right)$ on $\Delta_{\imath} \times \Delta_{j}$. Clearly $R_{N_{k}} \rightarrow R$ in the norm since

$$
\begin{aligned}
\left\|R_{N_{k}}-R\right\|^{2} & =\int_{0}^{T} \int_{0}^{T}\left|R_{N_{k}}-R\right|^{2} d t d \tau \\
& =\sum_{i, j=1}^{N} \int_{\Delta_{i} \times \Delta_{j}} \int\left|R\left(t_{i}, t_{j}\right)-R(t, \tau)\right|^{2} d t d \tau \\
& <\epsilon^{2} \sum_{i, j=1}^{N} \int_{\Delta_{i} \times \Delta_{j}} \int d t d \tau \\
& \leqq \epsilon^{2} T^{2} \rightarrow 0 \text { as } N_{k} \rightarrow \infty .
\end{aligned}
$$

The operator $R_{N_{k}}$ takes a function in $L^{2}(0, T)$ into a step function having constant values on the intervals $\Delta_{i}$. In particular a function $\phi_{N_{k}}$ which is piecewise constant:

$$
\phi_{N_{k}}=\phi_{i} \text { on } \Delta_{i}, \quad i=1, \cdots, N_{k}
$$

is carried over by $R_{N}$ into a step function

and

$$
\chi_{N_{k}}=R_{N_{k}} \phi_{N_{k}}
$$

$$
\chi_{N_{k}}=\chi_{j} \text { (constant) on } \Delta_{j}, \quad j=1, \cdots, N_{k} .
$$

For such a step function, the inverse operator exists (by virtue of our earlier assumptions) and

$$
\phi_{N_{k}}=R_{N_{k}}^{-1} \chi_{N_{k}}
$$

where

$$
\phi_{N_{k}}=\sum_{j=1}^{N_{k}} \frac{R_{N_{k}}^{-1}(i, j)}{\delta} \chi_{j} \text { on } \Delta_{i} \text {. }
$$

Also 


$$
\begin{aligned}
\left(R_{N_{k}}^{-1} \phi_{N_{k}}, \phi_{N_{k}}\right) & =\sum_{i, j=1}^{N_{k}} \frac{R_{N_{k}}^{-1}(i, j)}{\delta} \phi_{i} \phi_{j} \delta \\
& =\sum_{i, j=1}^{N_{k}} \phi_{i} R_{N_{k}}^{-1}(i, j) \phi_{j} .
\end{aligned}
$$

Now let $\phi$ be one of the regression functions in the original problem; let $\phi_{N_{k}}$ be the step function obtained from $\phi$ as follows:

$$
\begin{gathered}
\phi_{N_{k}}=\phi\left(t_{i}\right) \text { on } \Delta_{i}=\left(t_{i-1}, t_{i}\right), \\
\left|\Delta_{i}\right|=\delta_{i}=\delta,
\end{gathered} \quad\left(i=1, \cdots, N_{k}\right) .
$$

Then

$$
\left(R_{N_{k}} \phi_{N_{k}}, \phi_{N_{k}}\right)=\sum_{i, j=1}^{N_{k}} \phi\left(t_{i}\right) R_{N_{k}}\left(t_{i}, t_{j}\right) \phi\left(t_{j}\right) \delta^{2} .
$$

Using the spectral theorem for self-adjoint operators [1, pp. 188-190] we can write

$$
\begin{aligned}
\left(R_{N_{k}} \phi_{N_{k}}, \phi_{N_{k}}\right) & =\int_{-\infty}^{\infty} \lambda d_{\lambda}\left(\left(E_{\lambda}^{\left(N_{k}\right)} \phi_{N_{k}}, \phi_{N_{k}}\right)\right. \\
& =\sum_{j} \lambda_{j}^{\left(N_{k}\right)} \delta_{j}^{\left(N_{k}\right)}
\end{aligned}
$$

where $\lambda_{j}^{\left(N_{k}\right)}$ are the eigenvalues of the operator $R_{N_{k}}$ and $\delta_{j}^{\left(N_{k}\right)}$ are the (positive) jumps of bounded monotone nondecreasing function $\left(E_{\lambda}^{\left(N_{k}\right)} \phi_{N_{k}}, \phi_{N_{k}}\right)$. Similarly

$$
(R \phi, \phi)=\sum_{j} \lambda_{j} \delta_{j}
$$

where $\lambda_{j}, \delta_{j}$ have similar meanings w.r.t. $R$. Clearly

$$
\sum_{j} \lambda_{j}^{\left(N_{k}\right)} \delta_{j}^{\left(N_{k}\right)} \underset{N_{k} \rightarrow \infty}{\longrightarrow} \sum_{j} \lambda_{j} \delta_{j}
$$

Since $R_{N_{k}}^{-1}, R^{-1}$ are assumed to exist, and the functions $\phi_{N_{k}}, \phi$ are, respectively, in the domain of $R_{N_{k}}^{-1}, R^{-1}$,

$$
\begin{aligned}
\left(R_{N_{k}}^{-1} \phi_{N_{k}}, \phi_{N_{k}}\right) & =\sum_{j} \frac{1}{\lambda_{j}^{\left(N_{k}\right)}} \delta_{j}^{\left(N_{k}\right)}<\infty, \\
\left(R^{-1} \phi, \phi\right) & =\sum_{j} \frac{1}{\lambda_{j}} \delta_{j}<\infty .
\end{aligned}
$$


Since $R_{N_{k}} \rightarrow R$ in the norm the set of the eigenvalues of $R_{N}$ tends to the set of eigenvalues of $R$. Therefore

$$
\sum_{j} \frac{1}{\lambda_{j}^{N_{k}}} \delta_{j}^{\left(N_{k}\right)} \underset{N_{k} \rightarrow \infty}{\longrightarrow} \sum_{j} \frac{1}{\lambda_{j}} \delta_{j}
$$

provided the r.h.s. is convergent. But the r.h.s. is actually convergent since

$$
\left(R^{-1} \phi, \phi\right)=\sum \frac{1}{\lambda_{j}} \delta_{j}<\infty .
$$

Thus

$$
\left(R_{N_{k}}^{-1} \phi_{N_{k}}, \phi_{N_{k}}\right) \rightarrow\left(R^{-1} \phi, \phi\right)<\infty .
$$

This is true for each one of the regression functions. Hence the proposition. The continuity of $E_{T}$ in $T$ follows.

EXAmple. As an example consider the case of the purely nondeterministic process with spectral density $f(\lambda)=1 /\left(1+\lambda^{2}\right), r(t, \tau)=r(t-\tau)=e^{-|t-\tau|}$. In this case the linear integral operator $R$ with continuous kernel $r(t-\tau)$ $=e^{-|t-\tau|}$ is known to have an inverse $R^{-1}$ (see for instance [4, pp. 371-381]), viz. the differential operator

$$
1-\frac{d^{2}}{d t^{2}}
$$

plus boundary conditions at the end-points 0 and $T$. If the function $\phi(t)$ is sufficiently smooth, then

$$
\begin{aligned}
R^{-1} \phi(t)=\psi(\tau)=\phi(\tau) & -\phi^{\prime \prime}(\tau)+\left\{\phi(0)-\phi^{\prime}(0)\right\} r(\tau-0) \\
& +\left\{\phi(T)+\phi^{\prime}(T)\right\} r(\tau-T)
\end{aligned}
$$

To show that $R \psi(\tau)=\phi(t)$, we make use of Aronszajn's theory of a "reproducing kernel" Hilbert space [2]; that is, a Hilbert space of functions $f(x)$ defined on a set $E$ say, with a kernel $K(x, y)$ which satisfies the "reproducing" property:

$$
(f(x), K(x, y))_{x}=f(y)
$$

where the inner product on the left hand side is the inner product of the Hilbert space, $y$ being kept fixed. The class of functions $f(x)$ having the above property is generated by all the functions of the form $\sum_{k=1}^{n} \alpha_{k} K\left(x, y_{k}\right)$. The "norm" of such a linear combination is defined by the quadratic form

$$
\left\|\sum_{k=1}^{n} \alpha_{k} K\left(x, y_{k}\right)\right\|^{2}=\sum_{j, k=1}^{n} \alpha_{j} \bar{\alpha}_{k} K\left(y_{j}, y_{k}\right) .
$$


In our example, the kernel is $r(t-\tau)=e^{-|t-\tau|}$, the functions are continuous functions on $[0, T]$, and the inner product is the integral

$$
(\phi, \psi)=\int_{0}^{T} \phi(\tau) \overline{\psi(\tau)} d \tau .
$$

Now, if $\phi(t)$ is sufficiently smooth, then $\phi$ has the inverse image under $R$ :

$$
\begin{aligned}
R^{-1} \phi(t)=\psi(\tau)=\phi(\tau) & -\phi^{\prime \prime}(\tau)+\left\{\phi(0)-\phi^{\prime}(0)\right\} r(\tau-0) \\
& +\left\{\phi(T)+\phi^{\prime}(T)\right\} r(\tau-T)
\end{aligned}
$$

and hence $\phi$ is in the range-space of the operator $R$. It can be shown that a continuous function which is in the range-space of the operator $R$ has the "reproducing" property mentioned above. Thus, finally, for sufficiently smooth functions $\phi, \psi$ :

$$
\begin{aligned}
\left(R^{-1} \phi, \psi\right) \\
=\int_{0}^{T}\left[\phi(\tau)-\phi^{\prime \prime}(\tau)+\left\{\phi(0)-\phi^{\prime}(0)\right\} r(\tau-0)+\left\{\phi(T)+\phi^{\prime}(T)\right\} r(\tau-T)\right] \psi(\tau) d \tau \\
=\int_{0}^{T} \phi(\tau) \psi(\tau) d \tau+\int_{0}^{T} \phi^{\prime}(\tau) \psi^{\prime}(\tau) d \tau-\phi^{\prime}(T) \psi(T)+\phi^{\prime}(0) \psi(0) \\
\quad+\left\{\phi(0)-\phi^{\prime}(0)\right\} \int_{0}^{T} r(\tau-0) \psi(\tau) d \tau+\left\{\phi(T)+\phi^{\prime}(T)\right\} \int_{0}^{T} r(\tau-T) \psi(\tau) d \tau \\
=\int_{0}^{T} \phi(\tau) \psi(\tau) d \tau+\int_{0}^{T} \phi^{\prime}(\tau) \psi^{\prime}(\tau) d \tau-\phi^{\prime}(T) \psi(T)+\phi^{\prime}(0) \psi(0) \\
\quad+\left\{\phi(0)-\phi^{\prime}(0)\right\} \psi(0)+\left\{\phi(T)+\phi^{\prime}(T)\right\} \psi(T) \\
=\int_{0}^{T} \phi(\tau) \psi(\tau) d \tau+\int_{0}^{T} \phi^{\prime}(\tau) \psi^{\prime}(\tau) d \tau+\phi(0) \psi(0)+\phi(T) \psi(T) .
\end{aligned}
$$

The error-matrix $E_{T}$ is nonsingular in this case. This result agrees with the ones obtained by Mann and Moranda (Sankhya [8]) and by Grenander (Thesis [6]).

In the case of the general "elementary Gaussian" purely nondeterministic "THGM ${ }_{N}$ " process, we know that the integral operator $R$ has an inverse $R^{-1}$ (see for instance $[4$, appendix]). Aronszajn's theory of "reproducing" kernel Hilbert spaces can be used in this case also, and we conclude that in this case too, the error-matrix $E_{T}$ is nonsingular and continuous in $T$.

4. The approximation to the spectral density $f(\lambda)$ by spectral densities of discrete processes. Henceforth we shall use the discrete processes $D_{m}$ which are already used in the discussion ( $\$ 2$ ) of the least squares estimate: Let $n$ be a fixed positive integer. For each positive integer $m, D_{m}$ is defined to be the process $\left\{y_{k \epsilon_{m}}\right\}, k=0, \pm 1, \pm 2, \cdots, \epsilon_{m}$ being given by: $2^{m-1} n \epsilon_{m}=T$. 
We now want to consider the procedure of approximation to the given spectral density $f(\lambda)$ on $(-\infty, \infty)$ by a sequence of "discrete" spectral densities $f_{m}(\lambda)$ corresponding to the discrete processes $\mathfrak{D}_{m}$.

For a while we shall digress from our present problem and consider an arbitrary continuous parameter weakly stationary process $x(t),-\infty<t<\infty$, continuous in the mean. Let $F(\lambda)$ be the spectral distribution function of this process. Consider a sequence

$$
\cdots, z_{-1}, z_{0}, z_{1}, \cdots
$$

of independent positive stochastic variables, identically distributed. Although the discussion of this paragraph and the next two paragraphs is known (cf. $[7, \mathrm{pp} .58-59])$ it will be briefly repeated here for convenience. Put

$$
t_{\nu}=t_{\nu-1}+z_{\nu}, \quad \nu=\cdots,-1,0,1, \cdots .
$$

To determine the probability distribution of $t_{\nu}$, we can fix $t_{0}$ as 0 say, or we can let it have some arbitrary probability distribution. Then

$$
y_{\nu}=x\left(t_{v}\right), \quad \nu=\cdots,-1,0,1, \cdots
$$

is also stationary with covariances

$$
\begin{aligned}
E y_{\nu} \bar{y}_{\mu} & =E x\left(t_{\nu}\right) \bar{x}\left(t_{\mu}\right)=E\left[E x\left(t_{\nu}\right) \bar{x}\left(t_{\mu}\right) \mid t_{\nu}, t_{\mu} \text { fixed }\right] \\
& =E_{r_{t_{\nu}-t_{\mu}}}=E \int_{-\infty}^{\infty} \exp \left\{i\left(t_{\nu}-t_{\mu}\right) \lambda\right\} d F(\lambda) \\
& =\int_{-\infty}^{\infty} E \exp \left\{i\left(t_{\nu}-t_{\mu}\right) \lambda\right\} d F(\lambda) \\
& =\int_{-\infty}^{\infty}[\phi( \pm \lambda)]^{|\nu-\mu|} d F(\lambda)
\end{aligned}
$$

where $\phi(\lambda)=E e^{i \lambda z}$, the + or - sign being taken according as $\nu \geqq \mu$ or $\nu<\mu$.

To determine the spectrum of the new $y_{\nu}$-process define

$$
P_{\lambda}(a)=\int_{-\pi}^{\lambda} \frac{1-|a|^{2}}{\left|1-a e^{-i l}\right|^{2}} d l
$$

if $|a|<1$, and for $|a|=1, P_{\lambda}(a)$ is defined as the distribution function on $(-\pi, \pi)$ having all its mass at the point $\lambda=\theta$ where $a=e^{i \theta},-\pi<\theta \leqq \pi$. Thus for fixed $a(|a| \leqq 1) P_{\lambda}(a)$ is a distribution function in $\lambda(-\pi<\lambda \leqq \pi)$. Also

$$
\int_{-\pi}^{\pi} e^{i \nu \lambda} d P_{\lambda}(a)= \begin{cases}a^{\nu}, & \nu \geqq 0, \\ \bar{a}^{\nu}, & \nu<0 .\end{cases}
$$

Define the function $G(\lambda)$ as: 


$$
G(\lambda)=\int_{-\infty}^{\infty} P_{\lambda}[\phi(x)] d F(x)
$$

$G(\lambda)$ is well-defined since $|\phi(x)| \leqq 1$. Clearly $G(\lambda)$ is the spectral distribution junction of the $\left\{y_{\nu}\right\}$-process.

Let $z=h \xi$, where $E \xi=1$ and $h>0$ is a parameter, and consider the limiting behavior of $G(h \lambda)$ as the parameter $h \rightarrow 0$. The characteristic function of $G(h \lambda)$

$$
=\int_{-\pi / h}^{\pi / h} e^{i \nu \lambda} d \lambda G(h \lambda)=\int_{-\pi}^{\pi} e^{i y \lambda / h} d G(\lambda) .
$$

Then it is known (cf. [4, p. 59]) that

$$
\int_{-\pi / h}^{\pi / h} e^{i y \lambda} d \lambda G(h \lambda) \rightarrow \int_{-\infty}^{\infty} e^{i y x} d F(x)
$$

as $h \rightarrow 0$.

Now returning to our original problem of estimation, consider again the processes $\{y(t)\}$ and $\{x(t)\}$, and specialize the situation of the preceding paragraphs of this section as follows: The new assumptions $(\alpha)$ and $(\beta)$ on the spectral distribution function will be introduced progressively. Choose $t_{0}=0$, and $z=h \xi, h>0$ being a parameter and $\xi \equiv 1$ (or $\xi=1$ with probability 1). Then

$$
\phi(x)=E e^{i x z}=E e^{i x h \xi}=e^{i x h} .
$$

Let $P_{\lambda}(a)$ be as before, and consider for fixed $\lambda, P_{\lambda}(a)$ as a function of $a$. Now (with $a=e^{i 0}$ ) for $-\pi<\lambda \leqq \pi$

$$
P_{\lambda}\left(e^{i \theta}\right)= \begin{cases}0 & \text { if } \lambda<\theta, \\ 1 & \text { if } \lambda \geqq \theta .\end{cases}
$$

Therefore for fixed $\lambda(-\pi<\lambda \leqq \pi)$ and with $\phi(x)=e^{i x h}$

$$
P_{\lambda}[\phi(x)]=\left\{\begin{array}{l}
1, \quad \frac{-\pi+n 2 \pi}{h}<x \leqq \frac{\lambda+n 2 \pi}{h}, \\
0, \quad \frac{\lambda+n 2 \pi}{h}<x \leqq \frac{\pi+n 2 \pi}{h}
\end{array}\right.
$$

where $n$ is any integer positive, negative, or zero. The assumption $(\alpha)$ is as follows.

( $\alpha$ ) Now let $F(\lambda)$ the spectral distribution function of the $x$-process be absolutely continuous: $F(\lambda)=\int_{-\infty}^{\lambda} f(\mu) d \mu$, with $f(\mu) \geqq 0$ and $f \in L_{1}(-\infty, \infty)$. Thus in this special situation 


$$
\begin{aligned}
G(\lambda) & =\int_{-\infty}^{\infty} P_{\lambda}[\phi(x)] d F(x)=\int_{-\infty}^{\infty} P_{\lambda}[\phi(x)] f(x) d x \\
& =\sum_{n=-\infty}^{\infty} \int_{(-\pi+n 2 \pi) / h}^{(\lambda+n 2 \pi) / h} f(t) d t=\int_{-\pi}^{\lambda}\left\{\frac{1}{h} \sum_{n=-\infty}^{\infty} f\left(\frac{t+n 2 \pi}{h}\right)\right\} d t .
\end{aligned}
$$

Thus $G(\lambda)$ is seen to be absolutely continuous on $(-\pi, \pi)$ with spectral density

$$
G^{\prime}(\lambda)=\frac{1}{h} \sum_{n=-\infty}^{\infty} f\left(\frac{\lambda+n \cdot 2 \pi}{h}\right) .
$$

The series clearly converges for almost all $\lambda \in(-\pi, \pi)$. Now consider $G(h \lambda)$ with $h>0$. Clearly

$$
\begin{aligned}
d_{\lambda} G(h \lambda) & =h G^{\prime}(h \lambda) d \lambda=\left[\sum_{n=-\infty}^{\infty} f\left(\lambda+\frac{n 2 \pi}{h}\right)\right] d \lambda \\
& =f_{h}(\lambda) d \lambda
\end{aligned}
$$

say, with the notation: $f_{h}(\lambda)=\sum_{n=-\infty}^{\infty} f(\lambda+n \cdot 2 \pi / h)$. Thus in this special situation, the relation

$$
\int_{-\pi / h}^{\pi / h} e^{i y \lambda} d_{\lambda} G(h \lambda) \underset{h \rightarrow 0}{\longrightarrow} \int_{-\infty}^{\infty} e^{i y x} d F(x) \quad \text { for every } y,
$$

which was obtained earlier, becomes

$$
\int_{-\pi / h}^{\pi / h} e^{i y \lambda} f_{h}(\lambda) d \lambda \underset{h \rightarrow 0}{\longrightarrow} \int_{-\infty}^{\infty} e^{i \nu x} f(x) d \lambda \quad \text { for every } y .
$$

Now introduce the further assumption:

$(\beta)$ The spectral density $f(\lambda)$ is continuous and of bounded variation on $(-\infty, \infty)$.

The condition $(\beta)$ is, if we recall, a condition which ensures the validity of the Poisson summation formula (cf. Zygmund [9, p. 37]).

With these additional assumptions $(\alpha)$ and $(\beta)$, the following properties of the family of functions $\left\{f_{h}(\lambda)\right\}$ are established. (It will be understood that $h>0$.)

LEMMA 2. $f_{h}(\lambda)$ is periodic with period $2 \pi / h$, and further $\left\{f_{h}(\lambda)\right\}$ is a family of functions of uniformly bounded variation on any finite interval $(a, b)$. More precisely, given any finite interval $(a, b)$, there exists $h_{0}>0$ such that for $0<h$ $\leqq h_{0}, f_{h}(\lambda)$ is of uniformly bounded variation on $(a, b)$. Also for fixed $h>0, f_{h}(\lambda)$ is continuous.

The proof of this is just the proof of the validity of the Poisson summation formula (cf. Zygmund [9, p. 37]). 
LEMMA 3.

$$
\int_{-\pi / h}^{\pi / h} e^{i \nu \lambda} f_{h}(\lambda) d \lambda \underset{h \rightarrow 0}{\longrightarrow} \int_{-\infty}^{\infty} e^{i y x} f(x) d x \quad \text { for every } y .
$$

This has been established already.

LEMMA 4.

$$
\int_{-\pi / h}^{\pi / h} f_{\lambda}(\lambda) d \lambda<K, \quad K \text { being independent of } h .
$$

For, taking $y=0$ in Lemma 3, we obtain

$$
\int_{-\pi / h}^{\pi / h} f_{h}(\lambda) d \lambda=\int_{-\infty}^{\infty} f(\lambda) d \lambda<K .
$$

LEMMA 5. For any numbers $\alpha, \beta$ (fixed)

$$
\int_{\alpha}^{\beta}\left[f_{h}(\lambda)-f(\lambda)\right] d \lambda \rightarrow 0 \quad \text { as } h \rightarrow 0 .
$$

Proof. Since

$$
\int_{-\pi / h}^{\pi / h} f_{h}(\lambda) d \lambda=\int_{-\infty}^{\infty} f(\lambda) d \lambda
$$

therefore (remembering that $\int_{-\infty}^{\infty} f(\lambda) d \lambda<\infty$ )

$$
\int_{-\pi / h}^{\pi / h}\left[f_{h}(\lambda)-f(\lambda)\right] d \lambda=\int_{|\lambda| \geq \pi / h} f(\lambda) d \lambda \rightarrow 0 \quad \text { as } h \rightarrow 0 .
$$

Let $\alpha, \beta$ be fixed numbers and $h>0$ small enough so that $(\alpha, \beta) \subseteq(-\pi / h, \pi / h)$. Then

$$
0 \leqq \int_{\alpha}^{\beta}\left[f_{h}(\lambda)-f(\lambda)\right] d \lambda \leqq \int_{-\pi / h}^{\pi / h}\left[f_{h}(\lambda)-f(\lambda)\right] d \lambda \rightarrow 0 \quad \text { as } h \rightarrow 0 .
$$

Lemma 5 follows.

We now use the following simple result from the theory of Lebesgue integration: If $\phi_{n}(\lambda) \geqq 0$ for all $n$, on a measurable set $E$, and $\int_{E} \phi_{n}(\lambda) d \lambda \rightarrow 0$ as $n \rightarrow \infty$, then $\left\{\phi_{n}(\lambda)\right\}$ converges in measure to 0 (but it is not true that $\phi_{n}(\lambda) \rightarrow 0$ a.e.). Making use of this result, we immediately obtain the following.

Lemma 6. With the hypotheses introduced before the last lemma, there exists a sequence $\left\{h_{k}\right\}$ converging to 0 , such that $f_{h_{k}}(\lambda) \rightarrow f(\lambda)$ as $h_{k} \rightarrow 0$, for almost every $\lambda$. 
Next, just as a "collapsing" of $f(\lambda)$ to obtain $f_{h}(\lambda)$ was done above, so also a similar "collapsing" of the $Z(\lambda)$-process can be done. This, however, is not needed in the present context, and hence will not be discussed here.

5. The regression spectrum. Now it is necessary to state the assumptions about the regression functions. They are (1)-(5) below.

Assumptions about the $\phi$-functions:

(1) Let

$$
\Phi_{T}^{(r)}=\int_{\text {def. }}^{T}\left|\phi_{r}(t)\right|^{2} d t, \quad r=1,2, \cdots, p .
$$

Then $\Phi_{T}^{(r)} \rightarrow \infty$ as $T \rightarrow \infty, r=1, \cdots, p$.

(2) $\Phi_{T+h}^{(r)} / \Phi_{T}^{(r)} \rightarrow 1$ as $T \rightarrow \infty$, for any fixed $h, r=1, \cdots, p$.

$$
\operatorname{Lim}_{T \rightarrow \infty}\left(\int_{0}^{T} \phi_{r}\left(t+h \overline{\phi_{s}(t)} d t\right) /\left(\Phi_{T}^{(r)} \Phi_{T}^{(s)}\right)^{1 / 2}\right.
$$

exists for $h>0$ and $=R_{h}^{(r, s)}$, say; $1 \leqq r, s \leqq p$.

Certain consequences will follow from these assumptions, just as in the discrete case. To deal with negative $h$, first put $\phi_{r}(t)=0$ for $t<0$, and then define $R_{h}^{r, s}$ for all real $h$ by (3).

Now for $h \geqq 0$, substituting $u$ for $t-h$ and using the fact that $\phi_{r}(t)=0$ for $t<0$, we have

$$
\begin{aligned}
\frac{\int_{0}^{T} \phi_{r}(t-h) \overline{\phi_{s}(t)} d t}{\left(\Phi_{T}^{(r)} \Phi_{T}^{(s)}\right)^{1 / 2}} & =\frac{\int_{-h}^{T-h} \overline{\phi_{s}(u+h)} \phi_{r}(u) d u}{\left(\Phi_{T}^{(r)} \Phi_{T}^{(s)}\right)^{1 / 2}} \\
& =\frac{\int_{0}^{T} \overline{\phi_{s}(u+h)} \phi_{r}(u) d u}{\left(\Phi_{T}^{(r)} \Phi_{T}^{(s)}\right)^{1 / 2}} \\
& =\frac{\int_{0}^{T} \overline{\phi_{s}(u+h) \phi_{r}(u) d t}}{\left(\Phi_{T-h}^{(r)} \Phi_{T-h}^{(s)}\right)^{1 / 2}} \cdot \frac{\left(\Phi_{T-h}^{(r)} \Phi_{T-h}^{(s)}\right)^{1 / 2}}{\left(\Phi_{T}^{(r)} \Phi_{T}^{(s)}\right)^{1 / 2}} .
\end{aligned}
$$

Taking limits as $T \rightarrow \infty$ and using the "slowly increasing" character of $\Phi_{T}^{(r)}$ (see (2)) we obtain

$$
R_{-h}^{(r, s)}=\left[R_{h}^{(s, r)}\right]^{-} \text {; i.e., } R_{-h}=R_{h}^{*} \text { (the adjoint). }
$$

Next let $\alpha$ be an arbitrary column $p$-vector, and consider the quadratic form

$$
\left.\sigma_{\nu-\mu}=\alpha^{*} R_{\nu-\mu} \alpha=\lim _{T \rightarrow \infty} \sum_{r, s=1}^{p} \frac{\bar{\alpha}_{r}}{\left(\Phi_{T}^{(r)}\right)^{1 / 2}}\left[\int_{0}^{T} \phi_{r}(t+\nu) \overline{\phi_{s}(t+\mu}\right) d t\right] \frac{\alpha_{s}}{\left(\Phi_{T}^{(s)}\right)^{1 / 2}} .
$$

This relation is proved using the slowly increasing character of $\Phi_{T}^{(r)}$. Let $k$ be an $m$-vector, $m$ an arbitrary integer. Then 


$$
\sum_{\nu, \mu=1}^{m} \bar{k}_{\nu} \sigma_{\nu-\mu} k_{\mu}=\lim _{T \rightarrow \infty} \int_{0}^{T}\left|\sum_{\nu, r} \frac{\bar{\alpha}_{r} \bar{k}_{\nu}}{\left(\Phi_{T}^{(r)}\right)^{1 / 2}} \phi_{r}(t+\nu)\right|^{2} d t \geqq 0 .
$$

Hence $\alpha^{*} R \alpha$ is a non-negative-definite sequence. It follows that

$$
R_{h}=\int_{-\infty}^{\infty} e^{i h \lambda} d M(\lambda)
$$

where $M(\lambda)$ is a bounded nondecreasing hermitian matrix-valued function. Assume that $R_{0}=M(\infty)-M(-\infty)=M$ is nonsingular. The matrix function $M(\lambda)$ is called the spectral distribution function of the regression functions.

Now we introduce our next assumption about the regression functions.

(4) For any $\epsilon>0$, the corresponding "discrete" regression functions $\phi_{i}(n \epsilon)$, $n=0,1,2, \cdots ; i=1, \cdots, p$; satisfy the conditions of GR (see $[3$, pp. 233$235])$, and the spectral distribution function $M_{\epsilon}(\lambda)$ on $(-\pi / \epsilon, \pi / \epsilon)$ obtained asymptotically for this discrete situation (see [3]) converges to the spectral distribution function $M(\lambda)$ on $(-\infty, \infty)$, which is obtained in the continuous case (see (1)-(3)). The matrix distribution $d M(\lambda)$ will be assumed to have no mass outside a finite interval $(-A, A)$, and for $\pi / \epsilon \geqq A$, the distribution $d M_{\epsilon}(\lambda)$ is assumed to be the same as the distribution $d M(\lambda)$.

Next consider the discrete situations obtained from the continuous parameter situation by restricting our consideration to the values of the regression functions at the time points $k \epsilon, k=0, \pm 1, \pm 2, \cdots$, where $\epsilon$ is chosen to run through the sequence of values $\epsilon_{k}$ (tending to 0 ) such that $f_{\epsilon_{k}}(\lambda) \rightarrow f(\lambda)$ a.e. The existence of such a sequence has been established in Lemma 6 . The following lemma is now needed.

LEMma 7. Let the assumptions and the notation be as in the preceding paragraphs of this section. $f(\lambda)$ is assumed to be positive and continuous and of bounded variation on $(-\infty, \infty)$. $\left\{\epsilon_{k}\right\}$ tending to 0 is the sequence mentioned in the last paragraph. The integrals

$$
\begin{array}{ll}
\int_{-\pi / \epsilon_{k}}^{\pi / \epsilon_{k}} f_{\epsilon_{k}}(\lambda) d M_{\epsilon_{k}}(\lambda), & \int_{-\pi / \epsilon_{k}}^{\pi / \epsilon_{k}} \frac{1}{f_{\epsilon_{k}}(\lambda)} d M_{\epsilon_{k}}(\lambda), \\
\int_{-\infty}^{\infty} f(\lambda) d M(\lambda), & \int_{-\infty}^{\infty} \frac{1}{f(\lambda)} d M(\lambda),
\end{array}
$$

all exist. Contention: as $\epsilon_{k} \rightarrow 0$

$$
\begin{gathered}
\int_{-\pi / \epsilon_{k}}^{\pi / \epsilon_{\epsilon}} f_{\epsilon_{k}}(\lambda) d M_{\epsilon_{k}}(\lambda) \rightarrow \int_{-\infty}^{\infty} f(\lambda) d M(\lambda) . \\
\int_{-\pi / \epsilon_{k}}^{\pi / \epsilon_{k}} \frac{1}{f_{\epsilon_{k}}(\lambda)} d M_{\epsilon_{k}}(\lambda) \rightarrow \int_{-\infty}^{\infty} \frac{1}{f(\lambda)} d M(\lambda) .
\end{gathered}
$$


The proof of this lemma will be omitted. The last assumption about the regression functions is the following.

(5) For any $\epsilon_{k}$ as above, and corresponding $D_{k}(\$ 2)$, define as in [7, p. 235]

$$
D_{N_{k}, S_{k}}=\left[\begin{array}{ccc}
\left(\Phi_{N_{k}, S_{k}}^{(1)}\right)^{1 / 2} & & 0 \\
0 & \cdot & \left(\Phi_{N_{k}, S_{k}}^{(p)}\right)^{1 / 2}
\end{array}\right],
$$

the subscript " $S_{k}$ " having the same meaning as in $\$ 2$. For $D$ define

$$
D_{T}=\left[\begin{array}{ccc}
\left(\Phi_{T}^{(1)}\right)^{1 / 2} & & 0 \\
& \cdot & \\
0 & & \left(\Phi_{T}^{(p)}\right)^{1 / 2}
\end{array}\right] .
$$

We assume that

$$
\epsilon_{k}^{1 / 2} D_{N_{k}, S_{k}} D_{T}^{-1} \rightarrow I \text { (the identity matrix) }
$$

as $T \rightarrow \infty$, uniformly w.r.t. $\epsilon_{k}$.

6. Asymptotic expressions for the error-covariance matrices. We shall now derive asymptotic expressions (in the sense of Grenander and Rosenblatt) for the error-matrices $e_{T}$ and $E_{T}$, under the conditions $A$ (see Introduction).

Let $(0, T)$ be the interval over which the process $\left\{y_{t}\right\}$ is observed. Divide this interval $(0, T)$ into $n$ equal parts. Define the sequence $\left\{\epsilon_{k}\right\}$ by: $2^{k-1} n \epsilon_{k}$ $=T\left(=N_{k} \epsilon_{k}\right.$, say where $\left.N_{k}=2^{k-1} n\right)$. Out of this sequence $\left\{\epsilon_{k}\right\}$ there exists a subsequence, which again we shall denote by $\left\{\epsilon_{k}\right\}$, such that $f_{e_{k}}(\lambda) \rightarrow f(\lambda)$ a.e. (\$5), and henceforth we shall consider only this subsequence $\left\{\epsilon_{k}\right\}$. Denote by $D_{k}^{\prime}$ the discrete process $\left\{x\left(m \epsilon_{k}\right)\right\}, m=0, \pm 1, \pm 2, \cdots$. The process $D_{k}^{\prime}$ has the spectral density $g_{\epsilon_{k}}\left(\epsilon_{k} \lambda\right)$ on $\left(-\pi / \epsilon_{k}, \pi / \epsilon_{k}\right) . g_{\epsilon_{k}}(\lambda)$ is given by

$$
g_{\epsilon_{k}}(\lambda)=\frac{1}{\epsilon_{k}} \sum_{n=-\infty}^{\infty} f\left(\frac{\lambda+n 2 \pi}{\epsilon_{k}}\right) .
$$

From the spectral density $f(\lambda)$ on $(-\infty, \infty)$ was also constructed another spectral density $f_{\epsilon_{k}}(\lambda)$ of a distribution on $\left(-\pi / \epsilon_{k}, \pi / \epsilon_{k}\right)$. This was defined by

$$
f_{\epsilon_{k}}(\lambda)=\sum_{n=-\infty}^{\infty} f\left(\lambda+\frac{n 2 \pi}{\epsilon_{k}}\right),
$$

and

$$
\epsilon_{k} g_{\epsilon_{k}}\left(\epsilon_{k} \lambda\right)=f_{\epsilon_{k}}(\lambda)
$$


Now $f_{\epsilon_{k}}(\lambda)$ is continuous on $\left(-\pi / \epsilon_{k}, \pi / \epsilon_{k}\right)$. Also $f_{\epsilon_{k}}(\lambda)>0$ on $\left(-\pi / \epsilon_{k}, \pi / \epsilon_{k}\right)$. Therefore $g_{\epsilon_{k}}\left(\epsilon_{k} \lambda\right)$ is continuous and positive on $\left(-\pi / \epsilon_{k}, \pi / \epsilon_{k}\right)$. Thus both the spectral densities $f_{\epsilon_{k}}(\lambda), g_{\epsilon_{k}}\left(\epsilon_{k} \lambda\right)$ satisfy the conditions of [7] for the discrete case. For each $\epsilon_{k}$ and corresponding $D_{k}(\$ 2)$ and "discrete" regression functions $\phi_{i}\left(n \epsilon_{k}\right)$, define $D_{N_{k}, s_{k}}$ and $D_{T}$ as in the last section. Clearly $\epsilon_{k}^{1 / 2} D_{N_{k}, s_{k}}$ $\rightarrow D_{T}$ as $\epsilon_{k} \rightarrow 0$. Now for $D_{k}$ we have the asymptotic relations (cf. [7, pp. 237240]).

$$
\begin{aligned}
D_{N_{k}, S_{k}} e_{k} D_{N_{k}, S_{k}} & \sim M_{\epsilon_{k}}^{-1} 2 \pi \int_{-\pi / \epsilon_{k}}^{\pi / \epsilon_{k}} g_{\epsilon_{k}}\left(-\epsilon_{k} \lambda\right) d M_{\epsilon_{k}}(\lambda) M_{\epsilon_{k}}^{-1} \\
D_{N_{k}, S_{k}} E_{k} D_{N_{k}, S_{k}} & \sim\left[\frac{1}{2 \pi} \int_{-\pi / \epsilon_{k}}^{\pi / \epsilon_{k}} \frac{1}{g \epsilon_{k}\left(-\epsilon_{k} \lambda\right)} d M_{\epsilon_{k}}(\lambda)\right]^{-1}
\end{aligned}
$$

or

$$
\begin{aligned}
\epsilon_{k}^{1 / 2} D_{N_{k}, S_{k}} e_{k} D_{N_{k}, \epsilon_{k}} \epsilon_{k}^{1 / 2} & \sim M_{\epsilon_{k}}^{-1} 2 \pi \int_{-\pi / \epsilon_{k}}^{\pi / \epsilon_{k}} f_{\epsilon_{k}}(-\lambda) d M_{\epsilon_{k}}(-\lambda) M_{\epsilon_{k}}^{-1}, \\
\epsilon_{k}^{1 / 2} D_{N_{k}, S_{k}} E_{k} D_{N_{k}, S_{k}} \epsilon_{k}^{1 / 2} & \sim\left[\frac{1}{2 \pi} \int_{-\pi / \epsilon_{k}}^{\pi / \epsilon_{k}} \frac{1}{f_{\epsilon_{k}}(-\lambda)} d M_{\epsilon_{k}}(-\lambda)\right]^{-1}
\end{aligned}
$$

If we let $\epsilon_{k} \rightarrow 0$ in each term on the right side, we obtain, respectively:

$$
\begin{aligned}
& M^{-1} 2 \pi \int_{-\infty}^{\infty} f(-\lambda) d M(\lambda) M^{-1}, \\
& 2 \pi\left[\int_{-\infty}^{\infty} \frac{1}{f(-\lambda)} d M(\lambda)\right]^{-1} .
\end{aligned}
$$

Now we want to change the order of limits: we want to let $\epsilon_{k} \rightarrow 0$ first and then let $T \rightarrow \infty$, and show that we still obtain the same limit, in each case. The situation can be essentially explained by considering double sequences of numbers $\left\{a_{m n}\right\}$ and $\left\{b_{m n}\right\}$ such that

$$
\left.\begin{array}{rl}
a_{m n} b_{m n} & \underset{n}{\rightarrow} c_{m}>0 \\
a_{m n} \underset{n}{\rightarrow}
\end{array}\right\} \quad \text { for each } m,
$$

and

$$
0<c_{m} \underset{m}{\rightarrow} c>0
$$


Without loss of generality we can take $c_{m}=1$ for all $m$, and $c=1$. The condition (5) of $\$ 5$ amounts to the following

$$
\frac{a_{m n}}{a_{n}} \underset{n}{\rightarrow} \quad \text { uniformly w.r.t. } m \text {. }
$$

Then

$$
a_{m n} b_{m n} \underset{n}{\rightarrow} \quad \text { for each } m
$$

implies that

$$
a_{n} b_{m n} \underset{n}{\rightarrow} 1
$$

This means $a_{n} \sim 1 / b_{m n}$. However, the rate of growth of $a_{n}$ does not depend upon $m$ at all, i.e., is uniform w.r.t. $m$. Hence $a_{n} \sim 1 / b_{n}$, i.e.,

$$
a_{n} b_{n} \stackrel{n}{\rightarrow} 1 \text {. }
$$

This justifies the change in order of limits.

In terms of the matrices $D_{T}, e_{T}, E_{T}$, the last result amounts to the following:

LEMma 8. For any fixed $T_{0}>0$, and the sequence $\left\{m T_{0}\right\}_{m=1}^{\infty}$,

$$
\begin{aligned}
& \lim _{m \rightarrow \infty} D_{m T_{0}} e_{m} T_{0} D_{m T_{0}}=M^{-1} 2 \pi \int_{-\infty}^{\infty} f(-\lambda) d M(\lambda) M^{-1}, \\
& \lim _{m \rightarrow \infty} D_{m T_{0}} E_{m T_{0}} D_{m T_{0}}=2 \pi\left[\int_{-\infty}^{\infty} \frac{1}{f(\lambda)} d M(\lambda)\right]^{-1} .
\end{aligned}
$$

Here we note that $E_{T}, e_{T}, D_{T}$ are continuous functions of $T$ and that $D_{T} \rightarrow \infty$ as $T \rightarrow \infty$. Again, it is simpler to consider first functions $a_{T}$ and $b_{T}$ of a variable $T(0<T<\infty)$, where $a_{T}, b_{T}$ are both continuous functions of $T$, and $a_{T} \uparrow \infty$ as $T \rightarrow \infty$. The above lemma can be restated for such $a_{T}, b_{T}$ as:

LEмма 8. For any fixed $T_{0}>0$ and the corresponding sequence

$$
\left\{m T_{0}\right\}_{m=1}^{\infty}, \quad \lim _{m \rightarrow \infty} a_{m} T_{0} b_{m} T_{0}=1
$$

Now we want to prove that the last result implies:

$$
\lim _{T \rightarrow \infty} a_{T} b_{T}=1 .
$$

Now first let $\left\{T_{n}\right\}_{n=1}^{\infty}$ be a sequence $\ni T_{n} \uparrow \infty$.

Then for any $T_{n}, \exists$ integer $m \ni$ 


$$
m T_{0} \leqq T_{n}<(m+1) T_{0} .
$$

Let

$$
m T_{0}+h_{n}=T_{n}
$$

So

$$
\begin{aligned}
1 & =\frac{a_{m} T_{0}}{a_{m} T_{0}} \leqq \frac{a_{m} T_{0}+h_{n}}{a_{m} T_{0}}=\frac{a_{T_{n}}}{a_{m} T_{0}} \leqq \frac{a_{m} T_{0+m}}{a_{m} T_{0}} \\
& <1+\xi \text { say, where } \xi \rightarrow 0 . \\
\therefore \frac{a_{T_{n}}}{a_{m} T_{0}} & =1+\eta \text { where } \eta \rightarrow 0 \text { as } n \rightarrow \infty .
\end{aligned}
$$

This implies

$$
\frac{a_{T_{n}}}{a_{m T_{0}}} \rightarrow 1
$$

where the positive integer $m$ is as defined above. Or

$$
\frac{a_{m} T_{0}}{a_{T_{n}}} \rightarrow 1 .
$$

Similarly

$$
\frac{a_{(m+1) T_{0}}}{a_{T_{n}}} \rightarrow 1 .
$$

Thus

LEMMA 9.

$$
\begin{array}{r}
\lim _{T \rightarrow \infty} \frac{a_{T}}{a_{n} T_{0}}=1, \\
\lim _{T \rightarrow \infty} \frac{a_{T}}{a_{(n+1) T_{0}}}=1,
\end{array}
$$

where for any $T>0, n T_{0} \leqq T<(n+1) T_{0}$.

Now from Lemmas 8 and 9

Lemma 10. Taking any fixed $T_{0}>0$,

$$
\lim _{T \rightarrow \infty} a_{T} b_{n} T_{0}=1,
$$

where

$$
n T_{0} \leqq T<(n+1) T_{0} .
$$


Similarly

$$
\lim _{T \rightarrow \infty} a_{T} b_{(n+1) T_{0}}=1,
$$

where

$$
n T_{0} \leqq T<(n+1) T_{0}
$$

From this follows

LEMMA 11.

$$
\lim _{T \rightarrow \infty} a_{T} b_{T}=1
$$

Since $a_{T} b_{n T_{0}} \rightarrow 1$ therefore $a_{T} \sim 1 / b_{n T_{0}}$. However, the rate of growth of $a_{T}$ is independent of $T_{0}$, i.e., is uniform w.r.t. $T_{0}$. Hence $a_{T} \sim 1 / b_{T}$, i.e.,

$$
a_{T} b_{T} \underset{T \rightarrow \infty}{\longrightarrow} 1 \text {. }
$$

This proves our contention.

Thus finally we obtain

Theorem 2. Under the conditions A, as $T \rightarrow \infty$

$$
\begin{aligned}
& D_{T} e_{T} D_{T} \rightarrow M^{-1} 2 \pi \int_{-\infty}^{\infty} f(\lambda) d M(\lambda) M^{-1}, \\
& D_{T} E_{T} D_{T} \rightarrow 2 \pi\left[\int_{-\infty}^{\infty} \frac{1}{f(\lambda)} d M(\lambda)\right]^{-1} .
\end{aligned}
$$

7. Elements of the regression spectrum. The notions of "regression spectrum $S$ " and "elements of regression spectrum" are defined exactly as in the discrete case (see [7]), and, by exactly the same arguments as in the discrete case we obtain the following theorem. Our basic assumptions are the conditions $\mathrm{A}$ of the Introduction.

TheOREM 3. Let the assumptions be as in the past paragraph. Then the least squares estimate $c_{L, T}$ is asymptotically as efficient as the Markov estimate $c_{M, T}$ iff the spectral density is constant on each of the elements of $S$.

The class of regression functions mentioned above includes the following:

$$
\begin{array}{rlrl}
\phi(t) & =t^{\nu}, & \nu \geqq 0 \text { an integer; } \\
\phi(t)=t^{\nu} e^{i t \mu}, & \nu \geqq 0 \text { an integer; } \mu \text { any real number; } \\
\phi(t)=\left\{\begin{array}{ll}
t^{\nu} \cos t \mu \\
t^{\nu} \sin t \mu
\end{array},\right. & \nu \geqq 0 \text { an integer; } \mu \text { any real number. }
\end{array}
$$

The calculations are entirely analogous to those in the discrete parameter case (see [7, pp. 245-247]). 
8. Acknowledgments. The author is indebted to Professor M. Rosenblatt for suggesting the problem of this paper and for helpful criticism, and also to the referee for spotting some obscurities and for critical comments which led the author to think of the Theorem 1, §3. The paper was written at Indiana University during 1959-1960 and partly at Brookhaven National Laboratory during a summer appointment in 1959, and was revised at Columbia University. The author is indebted to these various institutions and the Office of Naval Research for financial support.

\section{REFERENCES}

1. N. I. Akhieser and I. M. Glasmann, Theorie der linearen Operatoren im Hilbert-Raum, Berlin, Akademie-Verlag, 1954.

2. N. Aronszajn, Theory of reproducing kernels, Trans. Amer. Math. Soc. vol. 68 (1950) pp. $337-404$

3. H. Cramér, On the theory of stationary random processes, Ann. of Math. vol. 41 (1940) pp. 215-230.

4. W. B. Davenport and W. L. Root, Random signals and noise, New York, McGraw-Hill, 1958, pp. 371-381.

5. J. L. Doob, The elementary Gaussian processes, Ann. Math. Statist. vol. 43 (1944) pp. 229-282.

6. U. Grenander, Stochastic processes and statistical inference, Ark. Mat. vol. I (1950) pp. 195-277.

7. U. Grenander and M. Rosenblatt, Statistical analysis of stationary time series, New York, J. Wiley and Sons, 1957.

8. H. B. Mann and P. B. Moranda, On the efficiency of the least squares estimates of parameters in the Ornstein-Uhlenbeck process, Sankhya vol. 13 (1953-1954) pp. 351-358.

9. A. Zygmund, Trigonometrical series, Warsaw, 1935.

INDIANA UNIVERSITY, BLOOMINGTON, Indiana

Columbia University,

NEW YORK, NEW YORK 